Pacific Journal of Mathematics

DEFINING RELATIONS FOR CERTAIN INTEGRALLY
PARAMETERIZED CHEVALLEY GROUPS 


\title{
DEFINING RELATIONS FOR CERTAIN INTEGRALLY PARAMETERIZED CHEVALLEY GROUPS
}

\author{
W. P. WARDLAW
}

For each faithful finite dimensional irreducible representation $R$ of a finite dimensional simple Lie algebra $L$ over the complex field, this paper treats the integrally parameterized subgroup $G_{Z}$ of the Chevalley Group $G$ over the rational field $Q$. For $L$ of type $A_{l}, D_{l}$, or $E_{l}$, Lie algebraic methods are used to extend a result of $\mathrm{J}$. Nielson on $S L(3, Z)$ to obtain a finite set of defining relations for $G_{Z}$. Similar relations augmented by defining relations for $G_{Z}\left(B_{2}\right)$ are shown to define $G_{Z}$ when $L$ is of type $B_{l}, C_{l}$, or $F_{4}$. (The relations for $G_{Z}\left(B_{2}\right)$ are not listed here.)

Defining relations for the $n$-dimensional group of lattice transformations have been given by W. Magnus in [4]. His method easily yields relations for the group $S L(n, Z)$ respectively $\operatorname{PSL}(n, Z)$ isomorphic to the universal respectively adjoint group $G_{Z}$ for $L$ of type $A_{n-1}$. H. Klingen [2] has proven the existence of a finite set of defining relations for $S p(2 n, Z)$, which is essentially the group $G_{Z}$ for $L$ of type $C_{n}$. Hence, the defining relations in $\S 2$ extend Magnus' result to $G_{Z}$ of types $D_{l}$ and $E_{l}$ and Klingen's result to $G_{Z}$ of types $B_{l}$ and $F_{4}$.

It might be helpful to the reader to note that a displayed equation is referred to by a symbol in parentheses, e.g., "(3.1)" or "(B)" and a theorem, lemma, or corollary is referred to by its title and a number without parentheses, e.g., "Lemma 3.1".

2. Statement of results. Let $R$ be a faithful finite dimensional irreducible representation of a finite dimensional simple Lie algebra $L$ over the complex field $C$, and let $\Sigma$ be the set of nonzero roots of $L$ with respect to some Cartan subalgebra. $L$ has a Chevalley basis $\left\{X_{\rho}\right.$, $\left.H_{\rho}: \rho \in \Sigma\right\}$ as defined in [1, p. 24, Th. 1] or [9, p. 6, Th. 1]. The $L$ module $V$ associated with $R$ contains a lattice $M$ which is invariant under the action of the Chevalley basis. If $M$ is properly chosen and $K$ is an arbitrary field, the automorphism $x_{\rho}(t)=\exp t R\left(X_{\rho}\right)$ on $V_{K}=$ $K \otimes_{z} M$ can be defined for each $\rho$ in $\Sigma$ and $t$ in $K$. The group $G_{K}$ generated by all of these automorphisms is the Chevalley group over the field $K$ of type $L$ corresponding to the representation $R . G_{K}$ is the adjoint respectively universal Chevalley group if $R$ is the adjoint respectively universal representation of $L$. (See [9, pp. 42-45].)

We will be concerned with the rational Chevalley group $G_{Q}$ (henceforth denoted by $G$ ) and its subgroup, the integrally parameterized Chevalley group $G_{Z}$ generated by the $x_{\rho}(t)$ with $\rho$ in $\Sigma$ and $t$ in $Z$. 
The relations $x_{\rho}(s) x_{\rho}(t)=x_{\rho}(s+t)$ in $G$ show the finite set $\left\{x_{\rho}(1): \rho \in\right.$ $\Sigma$ \} suffices to generate $G_{Z}$. Our goal is to find defining relations for $G_{Z}$ in terms of these generators.

We will let $P$ denote the set of positive roots and $I$, the set of simple roots with respect to some (henceforth fixed) regular ordering (as defined in [1, p. 20] or [9, p. 266]) of $\Sigma$. Greek letters $\alpha$ and $\beta$ will denote arbitrary simple roots, and $\gamma, \delta, \rho, \sigma$, and $\tau$ will be generic symbols for any roots.

A set $S$ of roots is called closed if $\rho, \sigma \in S$ and $\rho+\sigma \in \Sigma$ implies that $\rho+\sigma \in S$. A closed set $S$ of roots is an admissible system if $-\rho \in$ $S$ whenever $\rho \in S$. $S$ is a positive set of roots if $S$ is closed and $\rho \in S$ implies $-\rho \notin S$. If $S$ is a positive set of roots, it is possible to find a regular ordering of $\Sigma$ which makes all of the roots in $S$ positive; such a regular ordering will be called a relative ordering corresponding to the positive set $S$, to distinguish it from the fixed regular ordering. Finally, we define $L_{R}$ to be the additive group generated by the set of all weights of the representation $R$.

Consider the abstract generators $\left\{x_{\rho}: \rho \in \Sigma\right\}$ and define $w_{\rho}=x_{\rho} x_{-\rho}^{-1} x_{\rho}$ and $h_{\rho}=w_{\rho}^{2}$ for each $\rho \in \Sigma$. Designate the following relations:

$$
\left(\mathrm{A}^{\prime}\right) \quad\left(x_{\rho}, x_{\sigma}\right)=\Pi x_{i \rho+j \sigma}^{\gamma(i, j ; \rho, \sigma)}(\rho, \sigma \in \Sigma, \rho+\sigma \neq 0)
$$

where $(x, y)$ denotes the commutator $x y x^{-1} y^{-1}$ and the product is over all positive integers $i$ and $j$ such that $i \rho+j \sigma \in \Sigma$, taken in increasing order of the roots $i \rho+j \sigma$. The $C(i, j ; \rho, \sigma)$ are integers depending only on $i, j, \rho, \sigma$, the choice of Chevalley basis, and the structure of $L$. (See $[1$, p. 27] or $[9$, p. 22].)

$$
\begin{array}{lll}
\left(\mathrm{A}^{\prime \prime}\right) \quad w_{\rho} x_{\rho} w_{\rho}^{-1}=x_{-\rho}^{-1} & (\rho \in \Sigma) . \\
\text { (B) } \quad h_{\rho}^{2}=1 & (\rho \in \Sigma) \\
\text { (C) } \quad \Pi h_{\alpha}=1 \quad \text { if } \quad & I I(-1)^{c(\mu, \alpha)}=1 \text { for all } \mu \in L_{R},
\end{array}
$$

where $c(\beta, \alpha)$ is the Cartan integer $2(\beta, \alpha) /(\alpha, \alpha)$ and both products are over an increasing sequence $(\alpha)$ of (not necessarily all) simple roots. Let (A) denote the relations $\left(\mathrm{A}^{\prime}\right)$ respectively $\left(\mathrm{A}^{\prime \prime}\right)$ when $r k \quad \Sigma>1$ respectively $r k \Sigma=1$ ( $r k$ means $r a n k$ ). If $L$ is of type $A_{l}, D_{l}, E_{l}$, or $G_{2}$, let $(D)$ be the empty set of relations. If $L$ is of type $B_{l}, C_{l}$, or $F_{4}$, let $\alpha$ and $\beta$ be the simple roots forming a system of type $B_{2}$ with long root $\beta$, and let $G_{Z}(\alpha, \beta)$ be the subgroup of $G_{z}$ generated by $\left\{x_{\alpha}(1), x_{-\alpha}(1), x_{\beta}(1), x_{-\beta}(1)\right\}$. In this case, let (D) be the relations in $\left\{x_{\alpha}\right.$, $\left.x_{-\alpha}, x_{\beta}, x_{-\beta}\right\}$ obtained by replacing each $x_{\rho}(1)$ by $x_{\rho}$ in a set of defining relations for $G_{Z}(\alpha, \beta)$. The principal result is

MAIN THEOREM. Let $L$ be a finite dimensional simple Lie algebra over $C$ which is not of type $G_{2}$. Then the integrally parameterized 
Chevalley group $G_{Z}$ is isomorphic (by the canonical map defined by $\left.x_{\rho} \mapsto x_{\rho}(1)\right)$ to the abstract group $G^{\prime}$ generated by $\left\{x_{\rho}: \rho \in \Sigma\right\}$ subject to the relations $(\mathrm{A}),(\mathrm{B}),(\mathrm{C})$, and $(\mathrm{D})$.

The relations (C) can be omitted if $G$ is the universal Chevalley group. If $G$ is the adjoint Chevalley group, then $L_{R}=Z \Pi$ and can be replaced by $I I$ in the relations (C).

The main theorem is proved by showing (in $\S 4$ ) that a normal form for writing elements of $G_{Z}$ (given in Theorem 3.3) can be duplicated in the abstract group $G^{\prime}$.

In accomplishing the latter, it is shown that a set $E$ of compatible relations (See §4.) containing (A), (B), and (C) suffices to define $G_{Z}$ if it suffices to define the rank two subgroups of $G_{Z}$. This technique of parlaying defining relations for the rank two subgroups to defining relations for the whole group is reminiscent of Magnus' extension in [4] of Nielsen's result [6] and of Klingen's treatment in [2] of $S p(2 n, Z)$.

An explicit set of defining relations for $S p(4, Z)$ would probably allow the relations (D) for $L$ of type $B_{l}, C_{l}$, and $F_{4}$ to be explicitly stated, as suggested by $R$. Ree's identification $P S p(4, Q) \cong G_{Z}\left(C_{2}\right) \cong G_{Z}\left(B_{2}\right)$ in [7]. It seems likely that the relations (D) are in fact unnecessary.

3. A normal Form for $G$ and $G_{Z}$. In this section we develop several notions, notations, and a normal form in the concrete group $G_{Z}$ which we will use to study the abstract group in $\S 4$. Many of the results displayed in this section are known, and most of them appear in sources such as [1], [8], [9], and [10].

Let $U$ be the subgroup of $G$ generated by $\left\{x_{\rho}(r): \rho \in P, r \in Q\right\}$ and $U_{Z}$ the subgroup of $G_{Z}$ generated by $\left\{x_{\rho}(1): \rho \in P\right\}$. Corresponding to each root $\rho$ we define the one parameter subgroups $\mathfrak{X}_{\rho}=\left\{x_{\rho}(r): r \in Q\right\}$ of $G$ and $\mathfrak{X}_{\rho}(Z)=\left\{x_{\rho}(r): r \in Z\right\}$ of $G_{Z}$. More generally, for any $S \subseteq$ $\Sigma, \mathfrak{X}_{S}$ respectively $\mathfrak{X}_{S}(Z)$ is the subgroup of $G$ respectively $G_{Z}$ generated by $\left\{x_{\rho}(r): \rho \in S, r \in Q\right\}$ respectively $\left\{x_{\rho}(1): \rho \in S\right\}$. Then $G(\rho, \sigma, \cdots)=$ $\mathfrak{X}_{S}$ and $G_{Z}(\rho, \sigma, \cdots)=\mathfrak{X}_{S}(Z)$, where $S$ is the admissible system of roots generated by $\rho, \sigma, \cdots$.

Consider the homomorphism $\varphi_{\rho}$ from $S L(2, Q)$ into $G$ defined (See [1, pp. 33-37].) for each $\rho \in \Sigma$ by

$$
\varphi_{\rho}\left(\begin{array}{ll}
1 & 0 \\
r & 1
\end{array}\right)=x_{-\rho}(r), \varphi_{\rho}\left(\begin{array}{ll}
1 & r \\
0 & 1
\end{array}\right)=x_{\rho}(r) \quad(r \in Q) .
$$

$\varphi_{\rho}$ maps $S L(2, Q)$ onto $G(\rho)$. Its restriction to $S L(2, Z)$, which we also denote by $\varphi_{\rho}$, is a homomorphism into $G_{Z}$. Since $\left(\begin{array}{ll}1 & 0 \\ 1 & 1\end{array}\right)$ and $\left(\begin{array}{ll}1 & 1 \\ 0 & 1\end{array}\right)$ generate $S L(2, Z)$ (See [6, p. 8] or [3, vol. 2, Appendix B].), $\varphi_{\rho}$ maps $S L(2, Z)$ onto the subgroup $G_{Z}(\rho)$ of $G_{Z}$ generated by $x_{-\rho}(1)$ and $x_{\rho}(1)$. Now define 


$$
h_{\rho}(t)=\varphi_{\rho}\left(\begin{array}{ll}
t & 0 \\
0 & t^{-1}
\end{array}\right), w_{\rho}(t)=\varphi_{\rho}\left(\begin{array}{cc}
0 & t \\
-t^{-1} & 0
\end{array}\right),
$$

for each $\rho \in \Sigma$ and $t \in Q^{*}$, where $Q^{*}$ is the set of nonzero elements of Q. Then $H$ respectively $H_{Z}$ is the subgroup of $G$ respectively $G_{Z}$ generated by $\left\{h_{p}(t): \rho \in \Sigma, t \in Q^{*}\right\}$ respectively $\left\{h_{\rho}(-1): \rho \in \Sigma\right\}$, and $N$ respectively $N_{Z}$ is the subgroup of $G$ respectively $G_{Z}$ generated by $\left\{w_{\rho}(t): \rho \in \Sigma, t \in Q^{*}\right\}$ respectively $\left\{w_{\rho}(1): \rho \in \Sigma\right\}$.

The identities

$$
\begin{array}{ll}
w_{\rho}(t)=x_{\rho}(t) x_{-\rho}\left(-t^{-1}\right) x_{\rho}(t) & \left(\rho \in \Sigma, t \in Q^{*}\right), \\
h_{\rho}(t)=w_{\rho}(t) w_{\rho}(-1) & \left(\rho \in \Sigma, t \in Q^{*}\right),
\end{array}
$$

follow from corresponding identities in $S L(2, Q)$.

R. Steinberg showed in [9, p. 66 Th. 8 and p. 43 Lemma 28] (Also see [8].) that the group $G$ is defined by the generators $\left\{x_{\rho}(r): \rho \in \Sigma\right.$, $r \in Q\}$ subject to the relations

$$
\begin{aligned}
& x_{\rho}(r) x_{\rho}(s)=x_{\rho}(r+s) \\
& \left(x_{\rho}(r), x_{\sigma}(s)\right)=\Pi x_{\imath \rho+j \sigma}\left(C(i, j ; \rho, \sigma) r^{i} s^{j}\right) \quad(\rho+\sigma \neq 0) \\
& w_{\rho}(t) x_{\rho}(r) w_{\rho}(t)^{-1}=x_{-\rho}\left(-t^{2} r\right) \\
& h_{\rho}(t) h_{\rho}\left(t^{\prime}\right)=h_{\rho}\left(t t^{\prime}\right) \\
& \Pi h_{\alpha}\left(t_{\alpha}\right)=1 \text { if } I I t_{\alpha}^{e(\mu, \alpha)}=1 \text { for every } \mu \in L_{R},
\end{aligned}
$$

where $w_{\rho}(t)$ and $h_{\rho}(t)$ are defined by (3.3ab), $\rho, \sigma \in \Sigma, r, s \in Q, t, t^{\prime}, t_{\alpha} \in$ $Q^{*}$. The product $(3.4 b)$ is over all positive integers $i, j$ such that $i \rho+j \sigma$ is a root, taken in increasing root order, and the product in (3.4d) is taken over all $\alpha \in I I$ in increasing root order. The relations (3.4b) respectively $\left(3.4 b^{\prime}\right)$ can be omitted if $r k \Sigma=1$ respectively $r k \Sigma>1$, and the relations (3.4d) can be omitted if $G$ is the universal Chevalley group. If $R$ is the adjoint representation, it suffices to have $I I t_{\alpha}^{c(\mu, \alpha)}=1$ for all $\mu \in \| l$ in $(3.4 \mathrm{~d})$.

Let $W$ denote the Weyl group of $\Sigma$ and let $\omega_{\rho}$ denote the reflection in the hyperplane perpendicular to the root $\rho$. For $\rho, \sigma \in \Sigma, \sigma^{\prime}=\omega_{\rho}(\sigma)$, $c=c\left(\sigma^{\prime}, \rho\right), d=c(\sigma, \rho), t, s \in Q^{*}$, and $n=n(\rho, \sigma)= \pm 1$, the relations

$$
\begin{aligned}
& w_{\rho}(t) x_{\sigma}(s) w_{\rho}(-t)=x_{\sigma^{\prime}}\left(n t^{c} s\right), \\
& w_{\rho}(t) w_{\sigma}(s) w_{\rho}(-t)=w_{\sigma^{\prime}}\left(n t^{c} s\right), \\
& w_{\rho}(t) h_{\sigma}(s) w_{\rho}(-t)=h_{\sigma^{\prime}}\left(n t^{c} s\right) h_{\sigma^{\prime}}\left(n t^{c}\right)^{-1}, \\
& h_{\rho}(t) x_{\sigma}(s) h_{\rho}(t)^{-1}=x_{\sigma}\left(t^{d} s\right), \\
& h_{\rho}(t) w_{\sigma}(s) h_{\rho}(t)^{-1}=w_{\sigma}\left(t^{d} s\right), \\
& h_{\rho}(t) h_{\sigma}(s) h_{\rho}(t)^{-1}=h_{\sigma}\left(t^{d} s\right) h_{\sigma}\left(t^{d}\right)^{-1},
\end{aligned}
$$

hold in G. (See [1], [8, p. 119], or [9, p. 67] (3.5c) corrects a misprint in [8].) The last five relations are immediate from the first and the 
properties

$$
n(\rho, \sigma)=n(\rho,-\sigma)=(-1)^{c(\sigma, \rho)} n\left(\rho, \sigma^{\prime}\right), n(\rho, \rho)=-1,
$$

of the function $n: \Sigma \times \Sigma \rightarrow\{-1,1\}$, using (3.3ab).

Now it is clear from (3.5d) that $B=H U$ respectively $B_{z}=H_{Z} U_{Z}$ is a group containing $U$ respectively $U_{Z}$ as a normal subgroup, and, by [1, p. 42], $U \cap H=U_{Z} \cap H_{Z}=\{1\}$. An element $b$ of $B$ can be uniquely represented in the form

$$
b=h u
$$

with $h \in H$ and $u \in U$ expressed as

$$
\begin{array}{ll}
h=\Pi h_{\alpha}\left(t_{\alpha}\right) & \left(t_{\alpha} \in Q^{*}\right), \\
u=\Pi x_{\rho}\left(r_{\rho}\right) & \left(r_{\rho} \in Q\right),
\end{array}
$$

where the product in (3.7a) is over all $\alpha \in \Pi$ in increasing root order and the product in $(3.7 \mathrm{~b})$ is over all $\rho \in P$ in increasing root order. The expression (3.7b) is unique, and (3.7a) is unique modulo the relations (3.4d). (See [8, p. 122] and [1, p. 39, Lemme 6].) Moreover, by [9, p. 114, Lemma 49, and p. 115 Cor. 3], $B_{Z}=B \cap G_{Z}, H_{Z}=H \cap G_{Z}$, $U_{Z}=U \cap G_{Z}$, and an element $b=h u$ in the form (3.7ab) is in $B_{Z}$ if and only if each $t_{\alpha}= \pm 1$ and each $r_{\rho} \in Z$.

We will have use for the following easily proved result.

LEmma 3.1. Let the group $\mathfrak{X}$ be a product $\mathfrak{X}=\mathfrak{X}_{1} \cdots \mathfrak{X}_{n}$ of subgroups $\mathfrak{X}_{1}, \cdots, \mathfrak{X}_{n}$ such that $\left(\mathfrak{X}_{i}, \mathfrak{X}_{j}\right) \subseteq \Pi_{k \geqq j} \mathfrak{X}_{k}$ and uniqueness holds for the representation $x=x_{1} \cdots x_{n}\left(x_{i} \in \mathfrak{X}_{i}\right)$, and let $p$ be any permutation of the numbers $1,2, \cdots, n$. Then $\mathfrak{X}=\mathfrak{X}_{\left.p^{\prime} 1\right)} \cdots \mathfrak{X}_{p(n)}$ with uniqueness of representation.

Now if $S$ is a positive set of roots it follows from (3.4ab) that

$$
\mathfrak{X}_{S}=\Pi \mathfrak{X}_{\rho},
$$

where the product is over all $\rho \in S$ taken in increasing relative order. Since $\mathfrak{X}_{S} \subseteq U^{\prime}=\mathfrak{X}_{P^{\prime}}$ where $P^{\prime}$ is a positive system of roots containing $S$, the representation (3.7b) (for $U^{\prime}$ instead of $U$ ) of $u \in \mathfrak{X}_{S}$ is unique, so $(3.4 \mathrm{~b})$ and Lemma 3.1 show that any element $u \in \mathfrak{X}_{s}$ can be uniquely expressed in the form $u=\Pi x_{\rho}\left(r_{\rho}\right)$, with the product taken over all $\rho \in S$ in any fixed order.

There is a unique homomorphism $\zeta$ of $N$ onto the Weyl group $W$ of $L$, with kernel $H$, such that $w \cdot X_{\rho} \in Q \cdot X_{\omega(\rho)}$ when $\zeta(w)=\omega$. (Recall that $X_{\rho}$ is an element of the Chevalley basis of $L$, and that elements of $G$ act as automorphisms on $L$. See [1, p. 37, Lemma 3] or [9, pp. 29-31, Lemmas 20 and 22].) Thus $\psi: N / H \rightarrow W: H w \mapsto \zeta(w)$ is an 
isomorphism onto $W$. Moreover, for any $t \in Q^{*}, H w_{\rho}(t)=H w_{\rho}(1)$ and $\psi\left(H w_{\rho}(t)\right)=\zeta\left(w_{\rho}(1)\right)=\omega_{\rho}$. It is clear that $H_{Z}$ is the kernel of the restriction of $\zeta$ to $N_{Z}$ and $\zeta\left(N_{Z}\right)=W$. Thus $\psi_{Z}: N_{Z} / H_{Z} \rightarrow W: H_{Z} w \mapsto$ $\zeta(w)$ is an isomorphism onto $W$.

A set $N^{*}$ of representatives of $N$ modulo $H$ (as well as $N_{Z}$ modulo $H_{Z}$ ) can be chosen in $N_{Z}$ so that $w_{\rho}(1) \in N^{*}$ for each $\rho \in P$. For each $\omega \in W$ there is a unique representative $w(\omega) \in N^{*}$ such that $\zeta(w(\omega))=$ $\omega$. Henceforth, the elements $\omega \in W$ are frequently identified with the representatives $w(\omega)$, and both are denoted by $w$. We will also denote the reflection $\omega_{\rho}$ by $w_{\rho}$.

The Chevalley group $G$ has Bruhat decomposition

$$
G=\bigcup_{w \in W} B w B
$$

into disjoint double cosets $B w B=B w U_{w}$, where $U_{w}$ is the group generated by the $x_{\rho}$ such that $\rho>0$ and $w(\rho)<0$. This provides the normal form

$$
x=b w u \quad\left(b \in B, w \in N^{*}, u \in U_{w}\right)
$$

for uniquely expressing any element $x \in G$. Since (3.9) is invalid in $G_{Z}$ (since we might have $x=b w u \in G_{Z}$ with $b, u \notin G_{Z}$ ), we must modify this normal form to a normal form for $G$ which applies to $G_{Z}$ as well.

A reflection $w_{\alpha}$ in $W$ corresponding to a simple root $\alpha$ is called a simple reflection. It is well known that the simple reflections generate the Weyl group $W$. For each root $\rho$, let

$$
Y_{\rho}=\left\{\varphi_{\rho}\left(\begin{array}{ll}
a & b \\
c & d
\end{array}\right) \mid\left(\begin{array}{ll}
a & b \\
c & d
\end{array}\right) \in S L(2, Z), 0 \leqq a<c\right\},
$$

where $\varphi_{\rho}: S L(2, Q) \rightarrow G(\rho)$ is the canonical homomorphism described above (3.1). Then $Y_{\rho}$ is a system of representatives for $B \backslash B w_{\rho} B$, and we have

Lemma 3.2. For every $w \in W$ choose a minimal expression $w=$ $w_{\alpha} w_{\beta} \cdots w_{o}$ as a product of simple reflections. Then

$$
B w B=B Y_{\alpha} Y_{\beta} \cdots Y_{\delta}
$$

with uniqueness of expression on the right.

Lemma 3.2 is a special case of [9, pp. 99-100, Theorem 15 and Lemma 43]. (A more detailed proof for the special case was given in [10].)

For any rational number $r$, define

$$
x(r)=\left(\begin{array}{ll}
1 & r \\
0 & 1
\end{array}\right), y(r)=\left(\begin{array}{ll}
1 & 0 \\
r & 1
\end{array}\right), \text { and } \Omega=\left(\begin{array}{rr}
0 & 1 \\
-1 & 0
\end{array}\right) .
$$


Consider $A=\left(\begin{array}{ll}a & b \\ c & d\end{array}\right) \in S L(2, Z)$ with $0 \leqq a<c$. If $a=0$, then $c=1$, $b=-1$, and $A=y(-d) \Omega^{-1}$. If $a>0$, there is a positive integer $r_{1}$ such that $c=r_{1} a+c_{1}$ with $a>c_{1} \geqq 0$ and $d=r_{1} b+d_{1}$. If $c_{1}=0$, then $a=d_{1}=1$ and $A=y\left(r_{1}\right) x(b)$; if $c_{1}>0$, there is a positive integer $s_{1}$ such that $a=s_{1} c_{1}+a_{1}$ with $c_{1}>a_{1} \geqq 0, b=s_{1} d_{1}+b_{1}$, and $A=$ $y\left(r_{1}\right) x\left(s_{1}\right) A_{1}$ with $A_{1}=\left(\begin{array}{ll}a_{1} & b_{1} \\ c_{1} & d_{1}\end{array}\right) \in S L(2, Z)$ with $c>c_{1}>a_{1} \geqq 0$. By induction on $c$, repeated application of the division algorithm will yield

$$
A=y\left(r_{1}\right) x\left(s_{1}\right) \cdots y\left(r_{n-1}\right) x\left(s_{n-1}\right) y\left(r_{n}\right) x(k)
$$

or

$$
A=y\left(r_{1}\right) x\left(s_{1}\right) \cdots x\left(s_{n-1}\right) y\left(r_{n}\right) x\left(s_{n}\right) y(k) \Omega^{-1},
$$

where $n \geqq 0, r_{i}$ and $s_{i}$ are positive integers which do not appear if $n=0$, and $k$ is an integer. Clearly, the integers $n, r_{i}, s_{i}$, and $k$ are uniquely determined by $A$. In view of (3.1) and (3.2), transforming the above result by the homomorphism $\varphi_{\rho}$ shows that every element $g_{\rho}$ of $Y_{\rho}$ can be expressed in exactly one of the forms

$$
g_{\rho}=x_{-\rho}\left(r_{1}\right) x_{\rho}\left(s_{1}\right) \cdots x_{-\rho}\left(r_{n-1}\right) x_{\rho}\left(s_{n-1}\right) x_{-\rho}\left(r_{n}\right) x_{\rho}(k)
$$

or

$$
g_{\rho}=x_{-\rho}\left(r_{1}\right) x_{\rho}\left(s_{1}\right) \cdots x_{\rho}\left(s_{n-1}\right) x_{-\rho}\left(r_{n}\right) x_{\rho}\left(s_{n}\right) x_{-\rho}(k) w_{\rho}(-1),
$$

where the integers $n \geqq 0$, the positive integers $r_{i}$ and $s_{i}$ (which do not occur if $n=0$ ) and the integer $k$ are uniquely determined by $g_{\rho}$. Thus we have

THEOREM 3.3. For every $g \in G$, there is a unique $w \in W$ such that $g \in B w B$. Thus, for any minimal decomposition $w=w_{\alpha} w_{\beta} \cdots w_{r}$ of $w$ as a product of simple reflections $w_{\rho}, g$ can be expressed as a product of generators $x_{\rho}(r), h_{\delta}(t)$, and $w_{\delta}(1)(\rho \in \Sigma, \delta \in I)$ by writing

$$
g=b g_{\alpha} g_{\beta} \cdots g_{\gamma}
$$

with $b$ in the form (3.7ab), and each $g_{\delta} \in Y_{\delta}$ in one of the forms (3.11). Moreover, $g \in G_{Z}$ if and only if every parameter $r_{\rho}$ is an integer in (3.7b) and every parameter $t_{i}= \pm 1$ in (3.7a). Thus, (3.12) provides a normal form for $G_{Z}$.

4. The abstract group. In this section, we will consider several abstract groups generated by the symbols $x_{\rho}(\rho \in \Sigma)$ and defined by different sets of relations. A set $E$ of relations among the generators $x_{\rho}(\rho \in \Sigma)$ is called compatible if the corresponding relations in $G_{Z}$, obtained by replacing each $x_{\rho}$ by $x_{\rho}(1)$, are valid. Henceforth, $E$ will 
denote a compatible set of relations which contains the relations (A) of $\S 2$, and $G^{\prime}$ will denote the abstract group generated by the symbols $x_{\rho}(\rho \in \Sigma)$ and defined by the relations $E$. Thus the mapping $x_{\rho} \mapsto x_{\rho}(1)$ extends to an epimorphism $\pi: G^{\prime} \rightarrow G_{Z}$, which we call the canonical projection of $G^{\prime}$ onto $G_{Z}$.

For each $S=\left\{\sigma_{1}, \sigma_{2}, \cdots\right\} \subseteq \Sigma$, let $\mathfrak{X}^{\prime}(S)=\mathfrak{X}^{\prime}\left(\sigma_{1}, \sigma_{2}, \cdots\right)$ be the subgroup of $G^{\prime}$ generated by $\left\{x_{\sigma} \in G^{\prime}: \sigma \in S\right\}$, and let $G^{\prime}\left(\sigma_{1}, \sigma_{2}, \cdots\right)=$ $\mathfrak{X}^{\prime}(\bar{S})$, where $\bar{S}$ is the admissible system generated by $S$.

Lemma 4.1. Let $S$ be any positive set of roots. Then

$$
\mathfrak{X}^{\prime}(S)=I I \mathfrak{X}^{\prime}(\sigma),
$$

and any element $x \in \mathfrak{X}^{\prime}(S)$ can be expressed in the form

$$
x=\Pi x_{\sigma}^{k(\sigma)},
$$

where both products are taken over all roots $\sigma$ in $S$ in any (fixed) order, and $k(\sigma)$ are uniquely determined by $x$ and the order in which the product is taken.

Proof. First, induction on the cardinality $m$ of $S$ is used to show that $\mathfrak{X}^{\prime}(S)=\Pi \mathfrak{X}^{\prime}(\sigma)$ with uniqueness of expression, where the product is taken in increasing order (by some fixed relative ordering associated with $S$ ) of $\sigma \in S$. If $m=1$, this is clear. Suppose $m>1$. Let $\gamma$ be the smallest and $\delta$ the largest root of $S$ with respect to the relative ordering. Denote $S^{\prime}=S-\{\gamma\}, \mathfrak{X}^{\prime}(S)=\mathfrak{X}^{\prime}, \mathfrak{X}^{\prime}\left(S^{\prime}\right)=\mathfrak{X}^{\prime \prime} . \quad S^{\prime}$ is a positive set of roots with cardinality $m-1$, so the lemma holds for $\mathfrak{X}^{\prime \prime}$. Now, $\gamma+\delta>\delta$, so $\gamma+\delta \notin S$, and hence $\gamma+\delta \notin \Sigma$, since $S$ is closed. Since $S$ contains at least two linearly independent roots, $r k \Sigma>1$ and the relations $\left(A^{\prime}\right)$ hold in $G^{\prime}$. Thus $\left(x_{r}, x_{\delta}\right)=1$ and $x_{r}^{-1} x_{\hat{o}} x_{r}=x_{\delta} \in \mathfrak{X}^{\prime \prime}$. Suppose that for some $\sigma \in S^{\prime}, x_{\gamma}^{-1} x_{\rho} x_{\gamma} \in \mathfrak{X}^{\prime \prime}$ for every $\rho \in S^{\prime}$ with $\rho>\sigma$. Then $\left(x_{\gamma}, x_{\sigma}\right)=\Pi x_{\rho}^{n(\rho)}\left(\rho>\sigma, \rho \in S^{\prime}\right)$ is in $\mathfrak{X}^{\prime \prime}$, so $\left(x_{\sigma}, x_{\gamma}^{-1}\right)=x_{\gamma}^{-1}\left(x_{\gamma}, x_{\sigma}\right) x_{\gamma}=$ $I I\left(x_{\gamma}^{-1} x_{\rho} x_{\gamma}\right)^{n(\rho)}\left(\rho>\sigma, \rho \in S^{\prime}\right)$ is in $\mathfrak{X}^{\prime \prime}$. Hence $x_{\gamma} x_{\sigma} x_{\gamma}^{-1}=\left(x_{\gamma}, x_{\sigma}\right) x_{\sigma}$ and $x_{\gamma}^{-1} x_{\sigma} x_{\gamma}=\left(x_{\sigma}, x_{\gamma}^{-1}\right)^{-1} x_{\sigma}$ are in $\mathfrak{X}^{\prime \prime}$ for every $\sigma \in S^{\prime}$. Thus the elements of $\mathfrak{X}^{\prime}(\gamma)$ can be commuted to the left in any product of elements of $\mathfrak{X}^{\prime}$, leaving an element of $\mathfrak{X}^{\prime \prime}$ to the right, and the factors of this element can be taken in increasing root order by the induction hypothesis. Hence, the product $x=\Pi x_{\sigma}^{k(\sigma)}$ can be taken in increasing root order. Since the $k(\sigma)$ are uniquely determined in the image $\pi(x)=\Pi x_{\sigma}(k(\sigma))$ of $x$ under the canonical projection $\pi$, the $k(\sigma)$ are uniquely determined by $x$. (Note that this shows $\pi$ is biunique when restricted to $\mathfrak{X}^{\prime}(S)$.) The result for arbitrary root order now follows from Lemma 3.1. 
COROLlary 4.2. Let $S$ be a positive set of roots. Then the subgroup $\mathfrak{X}^{\prime}(S)$ of $G^{\prime}$ is isomorphic by the canonical projection $\pi$ to the corresponding subgroup $\pi\left(\mathfrak{X}^{\prime}(S)\right)=\mathfrak{X}^{\prime}(Z)$ of $G_{\%}$.

\section{COROLlary 4.3. The relations}

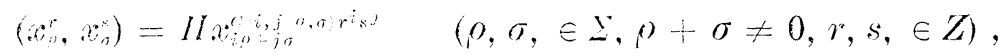

where the product is talien over all positive integers $i$ and $j$ such that in $+j \sigma \in \underline{Y}$ in increasing order of the roots io $+j \sigma$, hold in $G^{\prime}$.

Proof. $S=\left\{i_{\rho}-j \sigma \in \Sigma: i\right.$ and $j$ are nonnegative integers $\}$ is a positive set of roots, so the result follows from the isomorphism $\pi$ and the corresponding relations in $\mathfrak{t}_{S}(Z)$.

We now investigate various other relations in $G^{\prime}$. First, it will be useful to show the equivalence of three formulas $(4.3 \mathrm{abc})$. The definition $w_{\rho}=x_{\rho} x_{-\rho}^{-1} x_{\rho}(\rho \in \Sigma)$ gives the identity $w_{\rho} w_{-\rho}=w_{\rho} x_{\rho} w_{\rho}^{-1} x_{-\rho}$, and $h_{n}=w_{n}^{2}$ by definition, so the relation

$$
w_{\rho,} x_{,}, w_{p}^{-1}=x_{\cdots p}^{-1}
$$

is equivalent to

$$
w_{\rho} w_{-\rho}=h_{\mu} h_{-\rho}=1 \text {. }
$$

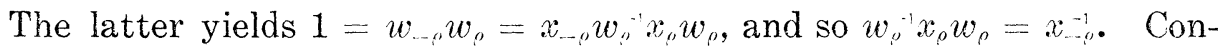
jugating this last relation or $(4.3 \mathrm{a})$ with appropriate powers of $w_{\rho}$ and using (4.3b) gives

$$
w_{\rho}^{t} x_{\sigma} w_{\rho}^{-t}=x_{\ldots \sigma}^{\cdots-1} \quad(\sigma= \pm \rho, t= \pm 1) .
$$

Clearly, for any $\rho \in \Sigma$, the relations (4.3abc) are equivalent. Moreover, the relation (4.3a) and the definition $h_{\sigma}=w_{\sigma}^{2}$ implies

$$
\left(w_{\sigma} x_{\sigma}\right)^{3}=h_{\sigma}^{2} \quad(\sigma= \pm \rho)
$$

We will now imitate the methods of [8] to discover more of the structure of $G^{\prime}$.

LEMMA 4.4. Let $\rho, \sigma \in \Sigma, \sigma^{\prime}=\omega_{\rho} \sigma, c=c\left(\sigma^{\prime}, \rho\right), t= \pm 1$, and $s \in Z$. Then

$$
w_{\rho}^{\dagger} x_{\sigma}^{s} w_{\rho}^{-t}=x_{\sigma^{\prime}}^{n(\rho, \sigma) t^{c} s}
$$

in $G^{\prime}$, where $n(\rho, \sigma)=n(\rho,-\sigma)= \pm 1$ depends only on $\rho$ and $\sigma$.

The proof of Lemma 4.4 is quite similar to the proof of Lemma 7.2 in [8, p. 118], and is hence omitted. It is perhaps helpful to note that when considering the case $\rho= \pm \sigma$ for the integrally parame- 
terized group, one uses the existence of roots $\gamma$ and $\delta$ such that $\left(x_{\gamma}\right.$, $\left.x_{\delta}\right)=x_{\rho}^{v} I l^{\prime}$ with $v= \pm 1$ occurs among the relations $\left(A^{\prime}\right)$.

Note that for any $\rho \in \Sigma$, (4.5) specializes to (4.3a), which implies that (4.3abc) and (4.4) hold in $G^{\prime}$.

Utilizing Lemma 4.4 and the definitions of $w_{\rho}$ and $h_{\rho}$, we get the next corollary.

COROLLARY 4.5. Let the notation be as in Lemma 4.4, and let $d=c(\sigma, \rho)$. Then the following relations hold in $G^{\prime}$ :

$$
\begin{aligned}
& w_{\rho}^{t} w_{\sigma}^{s} w_{\rho}^{-t}=w_{\sigma^{\prime}}^{n(\rho, \sigma) t} c_{s}, \\
& w_{\rho}^{t} h_{\sigma}^{s} w_{\rho}^{-t}=h_{\sigma^{\prime}}^{n(\rho, \sigma) t} c_{s}, \\
& h_{\rho}^{t} x_{\sigma}^{s} h_{\rho}^{-t}=x_{\sigma}^{(-1) d_{s}}, \\
& h_{\rho}^{t} w_{\sigma}^{s} h_{\rho}^{-t}=w_{\sigma}^{(-1) d_{s}}, \\
& h_{\rho}^{t} h_{\sigma}^{s} h_{\rho}^{-t}=h_{\sigma}^{(-1) d_{s}} .
\end{aligned}
$$

The next corollary is immediate from the relations $(B),(4.3 \mathrm{~b})$, (4.4), (4.7) and (4.10).

CoROLlary 4.6. Let the notation be as in Lemma 4.4. If the defining relations $E$ of $G^{\prime}$ contain the relations $(B)$, then the following relations hold in $G^{\prime}$ :

$$
\begin{aligned}
& h_{\rho}=h_{\rho}^{-1}=h_{-\rho}, \\
& \left(w_{\rho} x_{\rho}\right)^{3}=1, \\
& w_{\rho}^{t} h_{\sigma}^{s} w_{\rho}^{-t}=h_{\sigma^{\prime}}^{s}, \\
& h_{\rho} h_{\sigma}=h_{\sigma} h_{\rho} .
\end{aligned}
$$

We define $N^{\prime}$ (respectively $H^{\prime}$ ) to be the subgroup of $G^{\prime}$ generated by the elements $w_{\rho}$ (respectively $h_{\rho}$ ) for all roots $\rho \in \Sigma$. For each root $\rho, H^{\prime}(\rho)$ is the subgroup of $H^{\prime}$ generated by $h_{\rho}$. It is easy to show that $H^{\prime}$ is a normal subgroup of $N^{\prime}$ and that the mapping $H^{\prime} w_{\rho} \mapsto$ $\omega_{\rho}(\rho \in \Sigma)$ extends to an isomorphism of $N^{\prime} / H^{\prime}$ onto the Weyl group $W$, but we will not need this fact here.

Lemma 4.7. For any $\rho \in \Sigma, H^{\prime}(\rho)$ is a normal subgroup of $H^{\prime}$, and $H^{\prime}=I H^{\prime}(\alpha)$, where the product is taken over all simple roots $\alpha$. If $E$ contains the relations $(B)$, then $H^{\prime}$ is abelian.

The proof of Lemma 4.7 is omitted because of its similarity to the proof of $[8,7.7$, p. 120].

Now, let $U^{\prime}=\mathfrak{X}^{\prime}(P)$ be the subgroup of $G^{\prime}$ generated by $\left\{x_{\rho}: \rho \in P\right\}$ 
and let $B^{\prime}$ be the subgroup of $G^{\prime}$ generated by $H^{\prime} \cup U^{\prime}$.

Lemma 4.8. $U^{\prime} H^{\prime}=H^{\prime} U^{\prime}=B^{\prime}$ and $U^{\prime} \cap N^{\prime}=U^{\prime} \cap H^{\prime}=\{1\}$.

Proof. $\quad U^{\prime} H^{\prime}=H^{\prime} U^{\prime}=B^{\prime}$ by (4.8). Since $\pi\left(U^{\prime} \cap N^{\prime}\right)=U_{Z} \cap N_{Z}=\{1\}$ in $G_{Z}$ and $\pi$ is an isomorphism on $U^{\prime}$ by Corollary $4.2, U^{\prime} \cap N^{\prime}=\{1\}$ in $G^{\prime}$.

Corollary 4.9. Any element $b$ in $B^{\prime}$ can be uniquely decomposed in the form

$$
b=h u \quad\left(h \in H^{\prime}, u \in U^{\prime}\right) .
$$

Any $h \in H^{\prime}$ can be written in the form

$$
h=I I h_{\alpha}^{t(\alpha)} \quad(t(\alpha) \in Z),
$$

where the product is over all $\alpha \in \Pi$ in increasing root order. Any $u \in U^{\prime}$ can be written in the form

$$
u=\Pi x_{\rho}^{k(\rho)} \quad(k(\rho) \in Z),
$$

where the product is over all $\rho \in P$ in any order. The $k(\rho)$ in (4.15c) are uniquely determined by $u$ and the order in which the product is taken. Furthermore, the integers $t(\alpha)$ in $(4.15 b)$ can be chosen in $\{0$, $1\}$ if $E$ contains the relations $(B)$.

Proof. (4.15a), (4.15b), and (4.15c) are immediate from Lemmas $4.8,4.7$, and 4.1 , respectively.

CoROLlary 4.10. An element $b$ in $B^{\prime}$ is in the kernel of $\pi$ if and only if $b=I I h_{\alpha}^{t(\alpha)}$ with $I I(-1)^{t(\alpha) c(\mu, \alpha)}=1$ for every $\mu \in L_{R}$, where both products are taken over all $\alpha \in \Pi$ (in any order). If $E$ contains the relations $(B)$ and $(C)$, then $\pi$ restricted to $B^{\prime}$ is an isomorphism of $B^{\prime}$ onto $B_{Z}$.

Proof. Expressing $b$ in the form (4.15a), we have $\pi(b)=\pi(h) \pi(u)=$ 1 , so $\pi(u)$ lies in $U_{Z} \cap H_{Z}=\{1\}$. Thus $\pi(u)=1$ implies $u=1$ by Corollary 4.2, and $b=h$ can be written in the form (4.15b). Now, $\pi\left(h_{\rho}\right)=\pi\left(w_{\rho}^{2}\right)=w_{\rho}(1)^{2}=w_{\rho}(-1) w_{\rho}(-1)=h_{\rho}(-1)$, so $\pi(h)=$ $\Pi h_{\alpha}\left((-1)^{t(\alpha)}\right)=1$ if and only if $\Pi(-1)^{t(\alpha) c(\ell, \alpha)}=1$ for every $\mu \in L_{R}$, by (3.4d) and [9, p. 43, Lemma 28(c)]. If $E$ contains the relations (B) and (C), the relations (B) "reduce" the $t_{\alpha}$ to 0 or 1 , and then the condition for $b$ to be in the kernel of $\pi$ implies $b=1$ by the relations (C), so $\pi$ restricted to $B^{\prime}$ is an isomorphism.

Corollary 4.9 establishes a normal form for elements of $B^{\prime}$ which corresponds to the normal form in $B_{z}$ under the canonical projection $\pi$. Following the next technical lemma, we will seek to extend this 
normal form to all of $G^{\prime}$.

LEMma 4.11. Let $\rho, \sigma \in \Sigma$ be simple roots relative to some ordering of $\Sigma$. Then every element of $G^{\prime}(\rho)$ commutes with every element of $G^{\prime}(\sigma)$ if and only if $(\rho, \sigma)=0$.

Proof. Since $\rho, \sigma$ are simple roots in the relative ordering, $\rho-\sigma$ and $\sigma-\rho$ are not roots, and $\rho+\sigma$ is not a root if and only if $-q=$ $c(\rho, \sigma)=0$. Thus, $(\rho, \sigma)=0$ implies there are no roots $i \rho+j \sigma \epsilon$ $\Sigma(i, j \in Z)$ except $\pm \rho$ and $\pm \sigma$, so $\left(x_{\rho}, x_{\sigma}\right)=\left(x_{\rho}, x_{-\sigma}\right)=\left(x_{-\rho}, x_{\sigma}\right)=$ $\left(x_{-\rho}, x_{-\sigma}\right)=1$ by $\left(A^{\prime}\right)$. If $(\rho, \sigma) \neq 0,\left(x_{\rho}, x_{\sigma}\right) \neq 1$, since $\rho+\sigma$ is a root and the right hand product in $\left(A^{\prime}\right)$ has the factor $x_{o+\sigma}^{k}$ with $k=C(1,1$; $\rho, \sigma)= \pm 1$, and hence cannot be 1 because of the uniqueness in (4.15c).

We now try to duplicate the normal form of Theorem 3.3 for $G_{Z}$ in the abstractly defined group $G^{\prime}$. An element $x$ in $G^{\prime}$ such that $\pi(x) \in B w B$ is called completely decomposable (c.d.) if, for every minimal representation $w=w_{1} w_{2} \cdots w_{n}$ of $w$ in terms of simple reflections $w_{i}$, $x$ can be written in the normal form

$$
x=h u g_{1} g_{2} \cdots g_{n},
$$

where $h$ and $u$ are in the forms (4.15b) and (4.15c), respectively, and for each $i=1,2, \cdots, n$, either

$$
g_{i}=x_{-\alpha}^{r(1)} x_{\alpha}^{s(1)} \cdots x_{-\alpha}^{r(m)} x_{\alpha}^{i} \quad(m>0)
$$

or

$$
g_{i}=x_{-\alpha}^{r(1)} x_{a}^{s(1)} \cdots x_{a}^{\varsigma(m)} x_{-\alpha}^{k} w_{\alpha}^{-1} \quad(m \geqq 0),
$$

where $r(j)$ and $s(j)$ are positive integers, $k$ is an integer, and $\alpha$ is the simple root such that $w_{i}=w_{\alpha}$. A subset of $G^{\prime}$ is completely decomposable (c.d.) if every one of its elements is c.d. We denote by $Y_{\alpha}^{\prime}$ the set of all elements $g_{i}$ in $G^{\prime}(\alpha)$ which can be written in the form (4.16b) or (4.16c).

The expressions (4.16) are more conveniently treated in terms of the following generators of $G^{\prime}$.

Lemma 4.12. $G^{\prime}$ is generated by $\left\{x_{\alpha}, w_{\alpha} \in G^{\prime}: \alpha \in \Pi\right\}$.

Proof. Let $G^{*}$ be the subgroup of $G^{\prime}$ generated by $\left\{x_{\alpha}, w_{\alpha} \in G^{\prime}\right.$ : $\alpha \in \Pi\}$. For any $\rho \in P$, we show by induction on $h t(\rho)$ that $x_{\rho}$ and $x_{-\rho}$ are in $G^{*}$. If $h t(\rho)=1, \rho \in \Pi$ implies $x_{\rho}$ and $x_{-\rho}=w_{\rho} x_{\rho}^{-1} w_{\rho}^{-1}$ are in $G^{*}$. If $h t(\rho)>1$, there is a root $\alpha \in I I$ such that $\rho^{\prime}=w_{\alpha}(\rho)$ is a positive root with $h t\left(\rho^{\prime}\right)<h t(\rho)$. (See [9, p. 267 (10), (11)].) Thus, $x_{\rho^{\prime}}, x_{-\rho^{\prime}} \in G^{*}$ implies that $x_{\rho}=w_{\alpha} x_{\rho^{\prime}}^{n\left(\alpha, \rho^{\prime}\right)} w_{\alpha}^{-1}, x_{-\rho}=w_{\alpha} x_{-\rho^{\prime}}^{n\left(\alpha, \rho^{\prime}\right)} w_{\alpha}^{-1} \in G^{*}$ by 
(4.5). Since the $x_{\rho}(\rho \in \Sigma)$ generate $G^{\prime}, G^{*}=G^{\prime}$.

Now, the identity of $G^{\prime}$ is trivially c.d., and it follows inductively that $G^{\prime}$ is c.d. if it can be shown for each $x \in G^{\prime}$ that $x$ c.d. implies $x_{\alpha}^{t} x$ and $w_{n}^{t} x(\alpha \in I, t= \pm 1)$ are c.d. We use this to prove

Lemma 4.13. $G^{\prime}$ is c.d. if $G^{\prime}(\alpha, \beta)$ is c.d. for every $\alpha, \beta \in I i^{\prime}$.

Proof. Let $x \in \pi^{-1}(B w B)$ be c.d., let $\alpha$ be a simple root, and let $t= \pm 1$. For every minimal representation $w=w_{1} \cdots w_{n}, x$ can be put in the corresponding normal form (4.16) $x=h u g_{1} \cdots g_{n}$. Writing $x_{x}^{t} h u=h^{\prime} u^{\prime} \in B^{\prime}$ in the form (4.15), $x_{c}^{t} x=h^{\prime} u^{\prime} g_{1} \cdots g_{n}$ is in the form (4.16) corresponding to the minimal representation $w=w_{1} \cdots w_{n}$ of $w$, and $\pi\left(x_{n}^{i} x\right) \in B w B$. Thus, $x_{\alpha}^{t} x$ is c.d.

Now, consider $w_{a}^{+} x$. The normality of $H^{\prime}$ in $W^{\prime}$ implies $w_{a}^{+} H^{\prime}=$ $H^{\prime} w_{\alpha}^{t}=H^{\prime} w_{\alpha}^{-1}$, where we use $w_{\alpha}=h_{\alpha} w_{\alpha}^{-1}$ if $t=1$. By Lemma 4.1, any element $u \in U^{\prime}$ can be written in the form $u=\left(\| x_{p}^{k(p)}\right) x_{r}^{k}$, where the product $/ / x^{k, o}$ is taken over all $\rho \in P-\{\alpha\}$. Since $w_{\alpha}(P-\{\alpha\})=$ $P-\{\alpha\}$ (See [9, p. 267, (11)].), the relations (4.5) imply $u^{\prime}=w_{\kappa}^{-1}\left(I / x_{g^{\prime}}^{k(0)}\right) w_{r}$ is in $U^{\prime}$. Thus, $w_{\alpha}^{t} x=h^{\prime} u^{\prime} w_{\alpha}^{-1} x_{\alpha}^{k} g=h^{\prime} u^{\prime} x_{-\alpha}^{-k} w_{\alpha}^{-1} g$, with $h^{\prime}$ and $u^{\prime}$ in the forms (4.15b) and (4.15c), respectively, and $g$ in the form $g=g_{1} \cdots g_{n}$ is obtainable for every form $x=h u g_{1} \cdots g_{n}$ for $x$. Noting that $g_{n}=$ $x_{-\alpha}^{-k} w_{\alpha}^{-1}$ is in the form (4.16c), we see that $w_{\alpha}^{t} x=h^{\prime} u^{\prime} g_{\alpha} g_{1} \cdots g_{n}$ is in the normal form (4.16) if $w_{\alpha} w_{1} \cdots w_{n}$ is a minimal representation for $w_{\alpha} w$. If not, then there is a minimal representation for $w$ which begins with $w_{x}$. (See $[9$, p. 270, (21)].) Since $x$ is c.d., we can assume that $x=h u g_{1} \cdots g_{n}$ with $g_{1} \in Y_{\alpha}^{\prime}$. Since $G^{\prime}(\alpha)=G^{\prime}(\alpha, \alpha)$ is c.d. by hypothesis, we can write $g_{\alpha} g_{1}=h_{1} u_{1} g_{1}^{\prime}$ in the normal form (4.16) with $g_{1}^{\prime}=1$ or in the form $(4.16 \mathrm{~b})$ or $(4.16 \mathrm{c})$. In either case,

$$
w_{x}^{\prime} x=h^{\prime} u^{\prime} h_{1} u_{1} g_{1}^{\prime} g_{2} \cdots g_{n}=h^{\prime \prime} u^{\prime \prime} g_{1}^{\prime} g_{2} \cdots g_{n}
$$

can be put in the normal form (4.16), since both $w_{1} \cdots w_{n}$ and $w_{2} \ldots$ $w_{n}$ are minimal representations. The proof that $w_{\alpha}^{t} x$ is c.d. is completed in the following lemma, which shows that the decomposition (4.16) can be obtained corresponding to every minimal representation of the related Weyl group element.

Lemma 4.14. Let $G^{\prime}(\alpha, \beta)$ be c.d. for every $\alpha, \beta \in I /$. Suppose that $w=w_{1} \cdots w_{n}=w_{1}^{\prime} \cdots w_{n}^{\prime}$ are two minimal representations of $w \epsilon$ $W$, and that $g_{1}, \cdots, g_{n}$ are elements of $G^{\prime}$ such that $g_{2} \in Y_{\alpha}^{\prime}$ when $w_{i}=$ $w_{n}$. Then there exist $h \in H^{\prime}, u \in U^{\prime}$, and $g_{0}^{\prime}$ such that $g_{i}^{\prime} \in Y_{\alpha}^{\prime}$ when $w_{r}^{\prime}=w_{\alpha}$ and $g_{1} \cdots g_{n}=h u g_{1}^{\prime} \cdots g_{n}^{\prime}$.

Proof. By [5], $w_{1}^{\prime} \cdots w_{n}^{\prime}$ can be obtained from $w_{1} \cdots w_{n}$ by succes- 
sive substitutions of terms $w_{\alpha} w_{\beta} w_{\alpha} \cdots$ of $k$ factors by terms $w_{\beta} w_{\alpha} w_{\beta} \ldots$ of $k$ factors, where $k$ is the order of $w_{\alpha} w_{\beta}$, so it suffices to prove the lemma in the case

$$
w_{1} \cdots w_{j} w_{\alpha} w_{\beta} w_{\alpha} \cdots w_{j+k+1} \cdots w_{n}=w_{1} \cdots w_{j} w_{\beta} w_{\alpha} w_{\beta} \cdots w_{j+k+1} \cdots w_{n} \text {. }
$$

Since $G^{\prime}(\alpha, \beta)$ is c.d., we can write

$$
g_{j+1} \cdots g_{j+k}=h^{\prime} u^{\prime} g_{j+1}^{\prime} \cdots g_{j+k}^{\prime},
$$

with $g_{j+i}, g_{j+i+1}^{\prime} \in Y_{\alpha}^{\prime}$ and $g_{j+i}^{\prime}, g_{j+i+1} \in Y_{\beta}^{\prime}$ for odd integers $i$ between 1 and $k$.

Now we complete the proof by showing that the factor $h^{\prime} u^{\prime}$ can be "commuted" to the left to get

$$
g_{1} \cdots g_{n}=h u g_{1}^{\prime} \cdots g_{n}^{\prime},
$$

by showing that for any $\gamma \in \Pi, g_{\gamma} \in Y_{\gamma}^{\prime}, h^{\prime} \in H^{\prime}$, and $u^{\prime} \in U^{\prime}, g_{\gamma} h^{\prime} u^{\prime}=$ $h^{\prime \prime} u^{\prime \prime} g_{r}^{\prime}$ with $h^{\prime \prime} \in H^{\prime}, u^{\prime \prime} \in U^{\prime}$, and $g_{r}^{\prime} \in Y_{r}^{\prime}$. First, since $g_{r}$ is a product of powers of $x_{r}$ and $x_{-r}$, and $h^{\prime}$ is a product of $h_{\rho}(\rho \in \Sigma)$, we see by (4.8) that $g_{\gamma} h^{\prime}=h^{\prime} g$, where $g \in G^{\prime}(\gamma)$ can be written $g=h_{1} u_{1} g_{\gamma}^{\prime \prime}$ with $g_{\gamma}^{\prime \prime} \in Y_{\gamma}^{\prime}$, since $G^{\prime}(\gamma)$ is c.d. and $\pi(g) \in B w_{\gamma} B$.

Consider $u^{\prime}=x_{\gamma}^{s} \Pi_{\rho \neq \gamma} x_{\rho}^{k(\rho)}$. Since $G^{\prime}(\gamma)$ is c.d., we can write $\mathrm{g}_{i}^{\prime \prime} x_{\gamma}^{s}=$ $b_{1} g_{\gamma}^{\prime}$ with $b_{1} \in B^{\prime}$ and $g_{\gamma}^{\prime} \in Y_{\gamma}^{\prime}$. Let $\rho \in S=P-\{\gamma\}$. Then $p \rho \pm q \gamma \in S$ for any positive integers $p$ and $q$ such that $p \rho \pm q \gamma \in \Sigma$. Hence, $x=$ $\Pi_{\rho \in S} x_{\rho}^{k(\rho)} \in \mathfrak{X}^{\prime}(S)$ implies that $x_{ \pm \gamma}^{r} x x_{ \pm \gamma}^{-r}=\Pi_{\rho \in S}\left(x_{ \pm \gamma}^{r}, x_{\rho}^{k(\rho)}\right) x_{\rho}^{k(\rho)}$ is in $\mathfrak{X}^{\prime}(S)$ by (4.2). Since $g_{\gamma}^{\prime}$ is a product of powers of $x_{\gamma}$ and $x_{-r}$, it follows that $x^{\prime}=g_{r}^{\prime} x g_{\gamma}^{\prime-1} \in \mathfrak{X}^{\prime}(S) \subseteq U^{\prime}$.

Combining the above results and applying Corollary 4.9 to $h^{\prime} h_{1} u_{1} b_{1} x^{\prime} \in$ $B^{\prime}$, we obtain

$$
g_{r} h^{\prime} u^{\prime}=h^{\prime} h_{1} u_{1} b_{1} x^{\prime} g_{r}^{\prime}=h^{\prime \prime} u^{\prime \prime} g_{r}^{\prime}
$$

with $h^{\prime \prime} \in H^{\prime}, u^{\prime \prime} \in U^{\prime}$, and $g_{r}^{\prime} \in Y_{r}^{\prime}$. This completes the proof.

Now, if $G^{\prime}$ is c.d., $\pi$ gives a one-to-one correspondence between the normal form (4.16) in $G^{\prime}$ and the normal form of Theorem 3.3 in $G_{Z}$, modulo the subgroup $B^{\prime}$ of $G^{\prime}$. Thus the kernel of $\pi$ is the subgroup of $H^{\prime}$ described in Lemma 4.10, and, if $E$ contains the relations (B) and (C), then $\pi$ is an isomorphism of $G^{\prime}$ onto $G_{Z}$. We utilize this notion and Lemma 4.13 to complete the proof of the main theorem, by showing that each $G^{\prime}(\alpha, \beta)(\alpha, \beta \in \Pi)$ is c.d. when $E$ contains the relations $(B),(C)$, and $(D)$.

Case 1. $\alpha=\beta$ and $G^{\prime}(\alpha, \beta)=G^{\prime}(\alpha)$. By [6, p. 8] the group $S L(2$, $Z)$ is generated by $x=\left(\begin{array}{ll}1 & 1 \\ 0 & 1\end{array}\right)$ and $y=\left(\begin{array}{ll}1 & 0 \\ 1 & 1\end{array}\right)$ subject to the relations 


$$
y x^{-1} y x y^{-1} x=\left(x y^{-1} x\right)^{4}=1 .
$$

Substituting $x_{\alpha}$ for $x$ and $x_{-\alpha}$ for $y, w_{\rho}=x_{\rho} x_{-\rho}^{-1} x_{\rho}$, and $h_{\rho}=w_{\rho}^{2}$, these relations are

$$
w_{-\alpha} w_{\alpha}=h_{\alpha}^{2}=1,
$$

which hold in $G^{\prime}(\alpha)$ by $(4.3 \mathrm{~b})$ and (B). Since $S L(2, Z)$ is c.d. (by proof of Theorem 3.3) in terms of its generators $x$ and $y, G^{\prime}(\alpha)$ is also c.d.

Case 2. $\alpha, \beta \in I I,(\alpha, \beta)=0$. In this case, every element of $G^{\prime}(\alpha)$ commutes with every element of $G^{\prime}(\beta)$ by Lemma 4.11. Thus, $G^{\prime}(\alpha$, $\beta)=G^{\prime}(\alpha) G^{\prime}(\beta)$ and this case follows from Case 1 and the above commutativity property.

Case 3. $\alpha, \beta$ form a system of type $A_{2}$. The admissible set of roots generated by $\alpha$ and $\beta$ is $\Sigma_{0}=\{-\alpha-\beta,-\beta,-\alpha, \alpha, \beta, \alpha+\beta\}$, and $G^{\prime}(\alpha, \beta)$ is generated by $\left\{x_{\rho}: \rho \in \Sigma_{0}\right\}$. For this case, it can be shown that the structural constants satisfy $C(i, j ; \rho, \sigma)=0$ if $(i, j) \neq$ $(1,1), C(1,1 ; \rho, \sigma)=N_{\rho, \sigma}, N_{\rho, \sigma}=n(\rho, \sigma)$ if $\rho+\sigma \in \Sigma_{0}$, and $N_{\rho, \sigma}=0$ if $\rho+\sigma \notin \Sigma_{0}$, for every $\rho, \sigma \in \Sigma_{0}$. Moreover, $n(\alpha, \beta)=-n(\beta, \alpha)=n(\beta$, $\alpha+\beta)=-n(\alpha, \alpha+\beta)=n(\alpha+\beta, \beta)=-n(\alpha+\beta, \alpha), n(\rho, \rho)=n(\rho$, $-\rho)=-1$, and $n(\rho, \sigma)=n(\rho,-\sigma)=-n(-\rho, \sigma)$ for any $\rho, \sigma \in \Sigma_{0}$ with $\rho \neq \pm \sigma$. Thus, all of the constants are determined by the value of $n(\alpha, \beta)$. We assume $n(\alpha, \beta)=1$, renaming $\alpha$ and $\beta$, if necessary.

To parallel the notation of $[6, \S 2]$, we let the roots $\rho$ in $\Sigma_{0}$ correspond to subscripts $i j$ as follows:

$$
\begin{array}{rrrr}
\alpha \longleftrightarrow 12, \quad \beta \longleftrightarrow 23, & \alpha+\beta \longleftrightarrow 13, \\
-\alpha \longleftrightarrow 21, & -\beta \longleftrightarrow 32, \quad-\alpha-\beta \longleftrightarrow 31 .
\end{array}
$$

For $\rho \leftrightarrow i j$, we write $x_{\rho}=x_{i j}, w_{\rho}=w_{i j}$, and $h_{\rho}=h_{i j}$. Different letters will always denote different subscripts. In writing down the following relations, which are numbered to match corresponding relations from [6], we will put a reference to the relations implying them to the right. With this notation, the following relations hold in $G^{\prime}(\alpha, \beta)$ :

$\begin{array}{lll}\text { (A1) } & w_{i j}^{2}=h_{i j}=h_{j i} & w_{\rho}^{2}=h_{\rho} \text { and }(4.11) \\ \text { (A2) } & w_{i j} w_{j i}=1 & (4.3 \mathrm{~b}), \\ \text { (A3) } & w_{i j} w_{j k}=w_{j k} w_{i k} & (4.6), \\ \text { (A4) } & h_{i j}^{2}=1 & (4.11), \\ \text { (A5) } & h_{i j} h_{j k}=h_{j k} h_{i j} & (4.14), \\ \text { (A6) } & h_{12} h_{23} h_{13}=1 & (\mathrm{~A} 1),(4.9),(4.7),(\mathrm{A} 4), \\ \text { (B7) } & w_{i j}^{-1} x_{i j} w_{i j}=x_{j i}^{-1} & (4.5), \\ \text { (B8) } & w_{i k}^{-1} x_{i j} w_{i k}=x_{k j} & (4.5),\end{array}$



(B9) $\quad w_{j k}^{-1} x_{i j} w_{j k}=x_{i k}$
(B10) $h_{i j} x_{i j}=x_{i j} h_{i j}$
(B11) $h_{i k}^{-1} x_{i j} h_{i k}=x_{i j}^{-}$
(B12) $\quad h_{j k}^{-1} x_{i j} h_{j k}=x_{i j}^{-1}$
(C13) $w_{i j}^{-1} x_{i j} x_{j i}^{-1} x_{i j}=1$ $w_{\rho}=x_{\rho} x_{-,-1}^{-1} x_{\rho}$,
(C14) $\left(x_{i j}, x_{i k}\right)=1$
$\left(\mathrm{A}^{\prime}\right)$,
(C15) $\quad\left(x_{i j}, x_{k j}\right)=1$
(C16) $\quad\left(x_{i j}, x_{j k}\right)=x_{i k}$
$\left(\mathrm{A}^{\prime}\right)$.

The proof that these relations define $P S L(3, Z)=S L(3, Z)$ proceeds almost exactly as in $[6, \S 2]$. R. Ree has shown $P S L(3, Z) \cong G_{Z}\left(A_{2}\right)$ in [7]. Since one can obtain a "canonical" isomorphism of $G^{\prime}(\alpha, \beta)$ onto $G_{Z}\left(A_{2}\right)$, the group $G^{\prime}(\alpha, \beta)$ is c.d.

Case 4. $\alpha, \beta$ form a system of type $B_{2}$ with long root $\beta$. Then the relations (D) show that the "canonical" projection $\pi^{\prime}: G^{\prime}(\alpha, \beta) \rightarrow$ $G_{Z}\left(B_{2}\right)$ defined by $x_{\rho} \mapsto x_{\rho}(1)$ (in $G_{\zeta}\left(B_{2}\right)$ ) is an isomorphism onto the c.d. group $G_{Z}\left(B_{2}\right)$, and hence, $G^{\prime}(\alpha, \beta)$ is c.d.

This completes the proof of the main theorem.

\section{REFERENCES}

1. C. Chevalley, Sur certains groupes simples, Tohoku Math. J., (2), 7 (1955), 14-66.

2. H. Klingen, Charakterisierung der Siegelschen Modulgruppe durch ein endliches System definierender Relationen, Math. Annalen, 144 (1961), 64-82.

3. A. G. Kurosh, The theory of groups, 2nd ed., Chelsea Publishing Co., New York, 1960.

4. W. Magnus, Über n-dimensionalen Gittertransformationen, Acta Math., 64 (1934), 353-367.

5. H. Matsumoto, Générateurs et relations des groupes de Weyl généralisés, C. R. Acad. Sc. Paris, 258 (1964), 3419-3422.

6. J. Nielsen, Die Gruppe der dreidimensionalen Gittertransformationen, Det Kgl. Danske Videnskabernes Selskab. Math.-fysiske Meddelelser V, 12 (1924), 1-29.

7. R. Ree, On some simple groups defined by C. Chevalley, Trans. Amer. Math. Suc.. 84 (1957), 392-400.

8. R. Steinberg, Générateurs, relations, et revêtements de groupes algébriques, extrait du colloque sur la theorie des groupes algébriques, Centre Belge de Recherches Mathématiques, Louvain-Belgique (1962) 115-127.

9. R. Steinberg, Lectures on Chevalley Groups, Yale University Notes (1967).

10. W. Wardlaw, Defining relations for integrally parameterized Chevalley groups, doctoral dissertation, Univ. of California, Los Angeles (1966).

Received September 1, 1970. Most of the results in this paper are contained in the author's doctoral dissertation (UCLA, 1966), which was written while he was supported by the National Science Foundation Grant GP-3933. The author wishes to acknowledge the valuable guidance provided by Professor Robert Steinberg during the course of his research. 


\section{PACIFIC JOURNAL OF MATHEMATICS}

\section{EDITORS}

H. SAMELSON

Stanford University

Stanford, California 94305

C. R. HOBBY

University of Washington

Seattle, Washington 98105
J. DugundJI

Department of Mathematics

University of Southern California

Los Angeles, California 90007

RICHARD ARENS

University of California

Los Angeles, California 90024

\section{ASSOCIATE EDITORS}

E. F. BeCKENBACH

B. H. NeumanN

F. WOLF

K. YosHIDA

\section{SUPPORTING INSTITUTIONS}

UNIVERSITY OF BRITISH COLUMBIA

CALIFORNIA INSTITUTE OF TECHNOLOGY

UNIVERSITY OF CALIFORNIA

MONTANA STATE UNIVERSITY

UNIVERSITY OF NEVADA

NEW MEXICO STATE UNIVERSITY

OREGON STATE UNIVERSITY

UNIVERSITY OF OREGON

OSARA UNIVERSITY
UNIVERSITY OF SOUTHERN CALIFORNIA STANFORD UNIVERSITY

UNIVERSITY OF TOKYO

UNIVERSITY OF UTAH

WASHINGTON STATE UNIVERSITY

UNIVERSITY OF WASHINGTON

AMERICAN MATHEMATICAL SOCIETY

NAVAL WEAPONS CENTER

Printed in Japan by International Academic Printing Co., Ltd., Tokyo, Japan 


\section{Pacific Journal of Mathematics}

\section{Vol. 40, No. $1 \quad$ September, 1972}

Alex Bacopoulos and Athanassios G. Kartsatos, On polynomials

approximating the solutions of nonlinear differential equations........

Monte Boisen and Max Dean Larsen, Prüfer and valuation rings with zero

divisors ..........................................

James J. Bowe, Neat homomorphisms

David W. Boyd and Hershy Kisilevsky, The Diophantine equation

$$
u(u+1)(u+2)(u+3)=v(v+1)(v+2) \ldots \ldots \ldots \ldots \ldots \ldots \ldots
$$

George Ulrich Brauer, Summability and Fourier analysis ...............

Robin B. S. Brooks, On removing coincidences of two maps when only one,

rather than both, of them may be deformed by a homotopy ............

Frank Castagna and Geert Caleb Ernst Prins, Every generalized Petersen

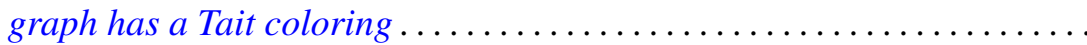

Micheal Neal Dyer, Rational homology and Whitehead products ..........

John Fuelberth and Mark Lawrence Teply, The singular submodule of a

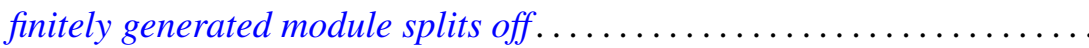

Robert Gold, $\Gamma$-extensions of imaginary quadratic fields ............ 83

Myron Goldberg and John W. Moon, Cycles in k-strong tournaments.......

Darald Joe Hartfiel and J. W. Spellmann, Diagonal similarity of irreducible

matrices to row stochastic matrices...............

Wayland M. Hubbart, Some results on blocks over local fields ..

Alan Loeb Kostinsky, Projective lattices and bounded homomorphisms....

Kenneth O. Leland, Maximum modulus theorems for algebras of operator

valued functions ...

Jerome Irving Malitz and William Nelson Reinhardt, Maximal models in the

language with quantifier "there exist uncountably many" ..

John Douglas Moore, Isometric immersions of space forms in space

forms.

Ronald C. Mullin and Ralph Gordon Stanton, A map-theoretic approach to

Davenport-Schinzel sequences ....................

Chull Park, On Fredholm transformations in Yeh-Wiener space. .

Stanley Poreda, Complex Chebyshev alterations ..............

Ray C. Shiflett, Extreme Markov operators and the orbits of Ryff. ...

Robert L. Snider, Lattices of radicals .....................

Ralph Richard Summerhill, Unknotting cones in the topological

category ................................

Charles Irvin Vinsonhaler, A note on two generalizations of $\mathrm{QF}-3 \ldots \ldots 229$

William Patterson Wardlaw, Defining relations for certain integrally

parameterized Chevalley groups...................

William Jennings Wickless, Abelian groups which admit only nilpotent

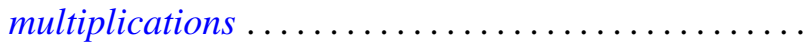

